This item was submitted to Loughborough's Research Repository by the author.

Items in Figshare are protected by copyright, with all rights reserved, unless otherwise indicated.

\title{
The biomechanical design of a training aid for a backward handspring in gymnastics
}

PLEASE CITE THE PUBLISHED VERSION

PUBLISHER

Springer / @ International Sports Engineering Association

VERSION

AM (Accepted Manuscript)

LICENCE

CC BY-NC-ND 4.0

\section{REPOSITORY RECORD}

Rosamond, Emma L., and Maurice R. Yeadon. 2019. "The Biomechanical Design of a Training Aid for a Backward Handspring in Gymnastics”. figshare. https://hdl.handle.net/2134/5875. 
This item was submitted to Loughborough's Institutional Repository (https://dspace.lboro.ac.uk/) by the author and is made available under the following Creative Commons Licence conditions.

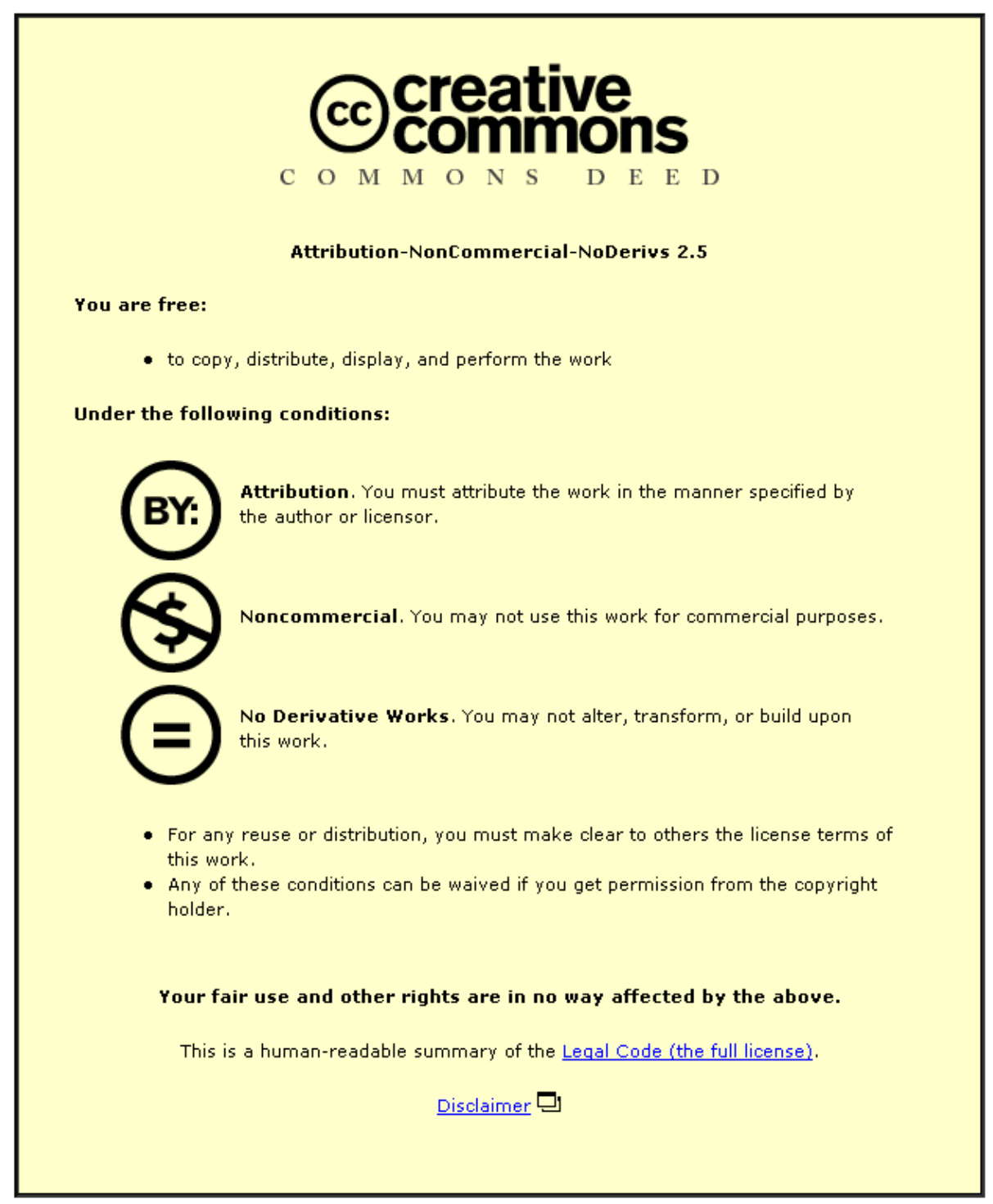

For the full text of this licence, please go to: http://creativecommons.org/licenses/by-nc-nd/2.5/ 


\title{
The biomechanical design of a training aid for a backward handspring in gymnastics
}

\author{
E.L. Rosamond ${ }^{1}$ and M.R.Yeadon ${ }^{2}$ \\ ${ }^{1}$ Wolfson School of Mechanical and Manufacturing Engineering, Loughborough University, \\ Leicestershire, LE11 3TU, UK. \\ ${ }^{2}$ School of Sport and Exercise Sciences, Loughborough University, Leicestershire, LE11 3TU, \\ UK.
}

\begin{abstract}
This study was carried out to design, construct and assess a training aid to assist in the learning of a backward handspring. A backward handspring is often the first backward dynamic skill gymnasts will learn and so its performance can be accompanied by anxiety. International high performance coaches were surveyed to establish the key coaching requirements of a backward handspring training aid. A video analysis of the skill was used to determine characteristic dimensions of the skill, and these were used in the design of the training aid. The aid was designed and manufactured in accordance with European Standards for safety. The device's safety for use in supporting the backward handspring was confirmed through testing. The assessment of the training aid using 11 gymnasts showed that it permitted a safe dynamic performance; provided support; did not obstruct technically good performances; allowed progressive use by novices without additional coach support; and was adjustable for gymnasts of various size and ability. When assessed against other training aids, it was the only aid that fulfilled all of the key coaching requirements.
\end{abstract}

\section{Introduction}

The backward handspring is a key skill in gymnastics and is frequently performed in six of the International Gymnastics Federation disciplines, namely: men's and women's artistic gymnastics, tumbling, acrobatic gymnastics, aerobics gymnastics and general gymnastics. The backward handspring involves the gymnast performing two connected flight phases, the first from the feet backwards onto the hands and the second from the hands onto the feet (Figure 1). The backward handspring can be used as an isolated skill, such as on the beam or in a floor routine, or as an accelerator skill in tumbling or vaulting.

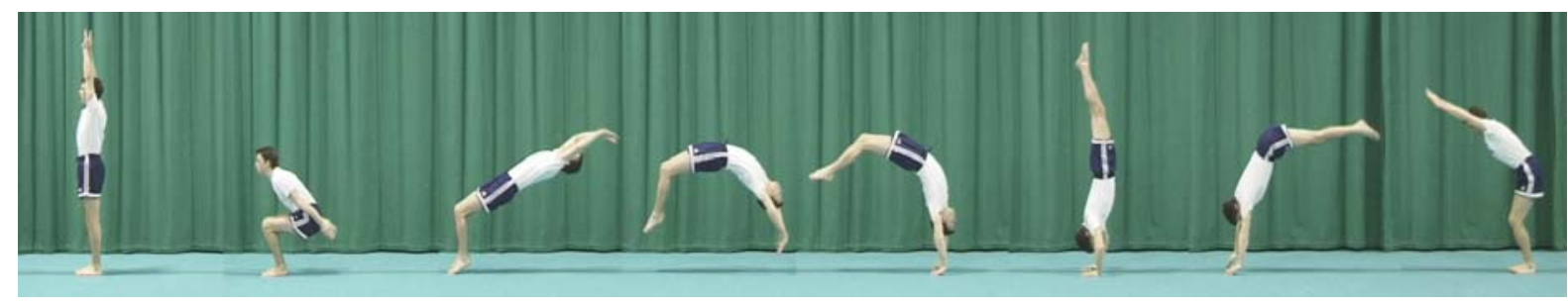

Figure 1. A standing backward handspring performance 
The backward handspring is often the first backward dynamic skill that a gymnast learns and, consequently, the initial learning stages may be accompanied by anxiety (Hayhurst, 1980). The backward handspring is usually learned from a two-footed standing position, and requires considerable manual assistance from the coach for correct shaping and support. The movement is a closed skill, which is modified on subsequent attempts via feedforward control on the basis of performance success (Holding, 1989). Consequently acquisition of this skill is reliant on high repetition of technically good performances. The progression from initial preparatory work to a technically good unsupported performance will generally take around two years. Common mistakes are incorrect head position and poor take-off technique (Mitchell et al., 2002), both of which can be a result of the gymnast being apprehensive, leading to insufficient travel in the first flight phase. Transmitted forces during hand contact may contribute to lateral compression injuries of the elbow joint (Koh et al., 1992) and as a consequence proper technique in the backward handspring is important in order to avoid injury (Sands and McNeal, 2006). A training aid that provided support, increasing safety and confidence, and also encouraged sufficient travel might help to overcome such problems. In this way aspects of poor take-off technique could be tackled, although incorrect head position would still need to be corrected.

Whilst training aids can be used in gymnastics to permit safe repetitions of preparatory skills without coach support, e.g. by lowering ground reaction forces as a coach's support does (Gervais et al., 2004), it should also allow development to perform the complete skill. The aims of this study were to establish the coaching requirements of a training aid for a backward handspring; to design a training aid to fit within the volume cleared by a backward handspring performance; and to assess a prototype with respect to the coaching requirements.

\section{Methods}

Initial interviews were conducted with 11 international high performance coaches to identify which skill might benefit most from a training aid. The backward handspring was the skill most selected by the coaches. Subsequent structured interviews were conducted to identify the key coaching requirements for such an aid. The requirements identified were:

- to permit a safe, dynamic performance;

- to support, but not impede the gymnast;

- to alleviate the requirement for support from the coach;

- to be adjustable for gymnasts of varying sizes and abilities;

- to allow progressive practice;

- to encourage good technique; and

- to build confidence and understanding.

This study was concerned with designing and assessing a prototype to achieve these requirements and so did not address all aspects, such as cost, durability, portability and ease of use, that apply to production runs. The safety requirement was achieved by designing the aid to European Standard EN913: 1996. Support during the performance depends largely on the dimensions of the volume of free space beneath the gymnast during the manoeuvre. Figure 2 shows superimposed images of a backward handspring, which define the basic shape and dimensions ( $\mathrm{a}, \mathrm{b}, \mathrm{c}$ and $\mathrm{d}$ see below) of the available space within which the aid must fit. 


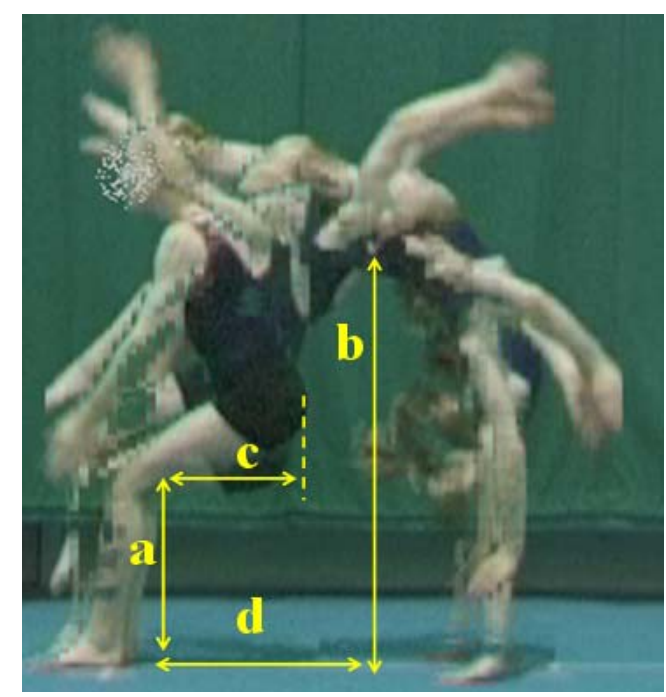

Figure 2. The four performance parameters: (a) sit height (vertical distance from the floor to the inside of the knee), (b) skill height (vertical distance from the floor to the highest point cleared), (c) sit length (horizontal distance from the heel to the rearmost body part in the sit position), (d) skill length (horizontal distance from the heel to the highest point cleared).

\subsection{Training aid dimensions determination}

In order to establish the required dimensions of the training aid, video data were collected in the National Gymnastics Performance and Research Centre at Loughborough University on 15 young gymnasts (age $=10.5 \pm 2.5$ years, height $=1.32 \pm 0.08 \mathrm{~m}$, mass $=$ $30.5 \pm 6.2 \mathrm{~kg}$ ), performing a two-footed standing backward handspring. The data were collected using a digital video camera recording at 50 fields per second, located $8.75 \mathrm{~m}$ from the gymnast with its axis perpendicular to the plane of movement. Video recordings were made of calibration poles with markers in known locations placed in the plane of the performance and these were manually digitised to determine horizontal and vertical scale factors, which were used to obtain spatial measurements from the manual video digitisation of the backward handspring performances.

Each gymnast's standing height $h$ was measured and four performance parameters (sit height $a$, sit length $c$, skill height $b$, skill length $d$ ), which defined the free space beneath the skill (Figure 2), were calculated from the video data. A linear relationship was assumed to hold between the four parameters $a, b, c$ and $d$ and the standing height $h$ as sit height $(a)$ and sit length $(c)$ are likely to be related to shank and thigh length, whilst skill height $(b)$ and length $(d)$ are likely to be greater for taller gymnasts. These regression equations (Table 1$)$ were then used in the design of an adjustable prototype training aid so that it fit within the volume cleared by a backward handspring for gymnasts with standing heights between $1.1 \mathrm{~m}$ and $1.7 \mathrm{~m}$ (Figure 3). 


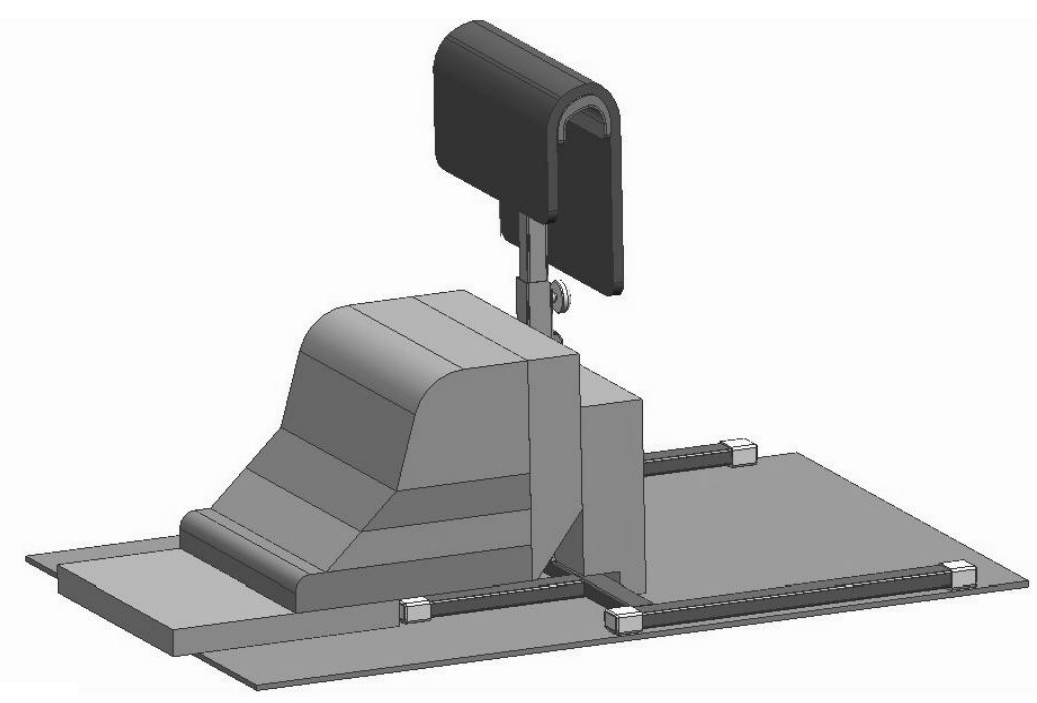

Figure 3. The training aid design. The box section frame was made of steel with an adjustable vertical section and standard gymnastics fittings and was covered with standard gymnastics foams to cushion any contact.

Table 1: Linear regressions of performance parameters against standing height

\begin{tabular}{|l|c|c|c|c|c|}
\hline \multicolumn{1}{|c|}{ parameter } & $m$ & $k$ & $\mathrm{R}^{2}$ & $\mathrm{p}$ & $\begin{array}{c}\text { standard } \\
\text { error }\end{array}$ \\
\hline$a$ - sit height & 0.461 & -308 & 0.63 & $<0.01$ & $28 \mathrm{~mm}$ \\
\hline$b-$ skill height & 1.026 & -610 & 0.89 & $<0.01$ & $29 \mathrm{~mm}$ \\
\hline$c$ - sit length & 0.208 & 38 & 0.24 & 0.06 & $30 \mathrm{~mm}$ \\
\hline$d-$ skill length & 0.481 & -153 & 0.35 & 0.02 & $52 \mathrm{~mm}$ \\
\hline
\end{tabular}

Note: $\mathrm{m}$ and $\mathrm{k}$ are coefficients of regression equation $x=m h+k$ for parameter $x$ and height $h$ (in $\mathrm{mm}$ )

The $\mathrm{R}^{2}$ value, the significance level $\mathrm{p}$, and the standard error of fit were calculated for the linear regression of each performance parameter against standing height (Table 1). While the $R^{2}$ values were quite high for sit height and skill height (Figure 4), indicating that the linear assumption was valid, the values were relatively low for sit length and skill length as evidenced by the greater scatter in Figure 5. Despite the reduced linearity of the dependence on gymnast height these parameters had standard errors of fit for sit length and skill length of only $0.03 \mathrm{~m}$ and $0.05 \mathrm{~m}$ respectively. Thus, at a given setting the adjustable prototype was able to accommodate performance variations of up to $\pm 0.1 \mathrm{~m}$ using three settings to cover a gymnast height range of $1.1 \mathrm{~m}-1.7 \mathrm{~m}$. The regression equation for skill height provides a guide for the setting to be chosen based upon gymnast standing height. All tolerances of the aid were designed to fit within the volume cleared by the skill to ensure that the aid would not interact with the gymnast when the skill was performed well, but would support the gymnast during a poor performance. 


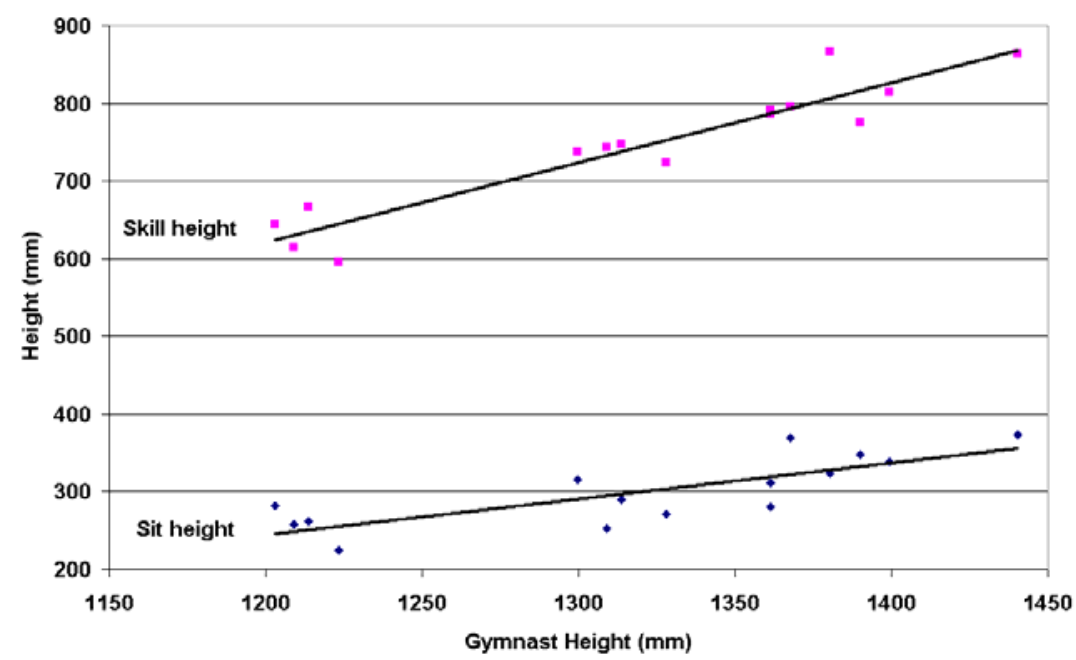

Figure 4. Regression lines for sit height and skill height against standing height.

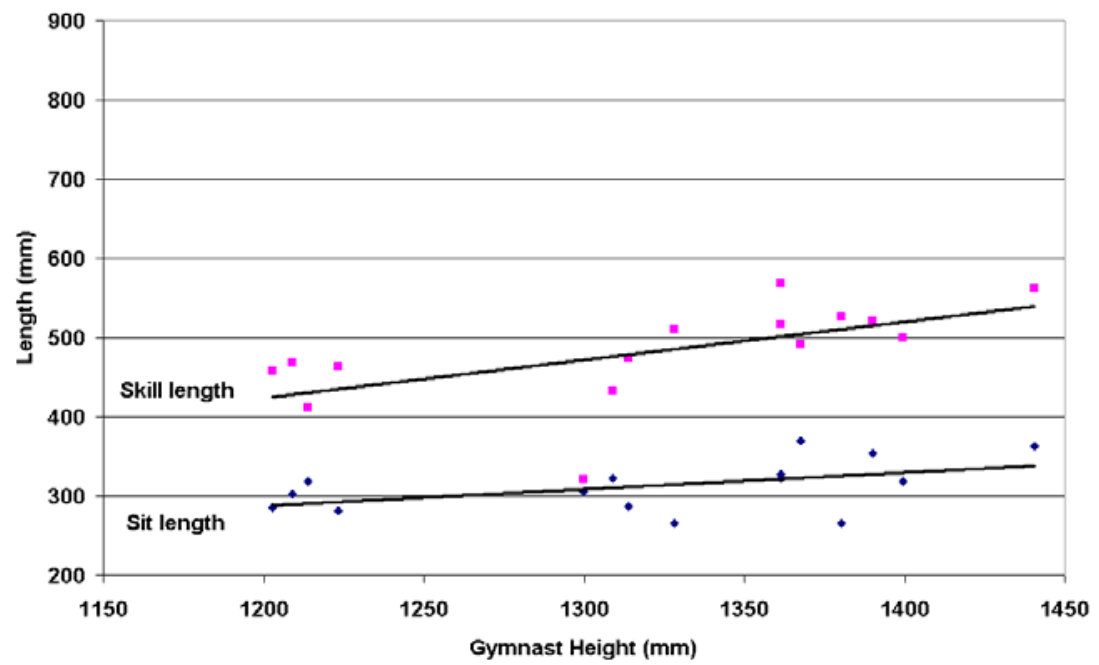

Figure 5. Regression lines for sit length and skill length against standing height.

With the training aid dimensions determined, methods such as collision analysis were carried out by mapping the image of a performed skill (e.g. Figure 2) over the image of the training aid (e.g. Figure 3) to assess the performance of the aid against the key design requirements.

\subsection{Fabrication}

The prototype required a stiff and rigid frame, which was constructed from $2 \mathrm{~mm}$ thick welded steel box section $(50 \mathrm{~mm} \times 30 \mathrm{~mm}$ for the horizontal sections, $45 \mathrm{~mm}$ x $45 \mathrm{~mm}$ and $40 \mathrm{~mm} \times 40 \mathrm{~mm}$ for the adjustable vertical column). The horizontal support section at the top of the vertical column was covered with $70 \mathrm{~mm}$ high density closed cell polyurethane foam $\left(38 \mathrm{~kg} \mathrm{~m}^{-3}\right)$ for impact with $30 \mathrm{~mm}$ lower density foam $\left(20 \mathrm{~kg} \mathrm{~m}^{-3}\right)$ on top for comfort to ensure that suitably low impact forces were achieved to meet European Standard EN913: 1996. The resulting prototype had a mass of $21.7 \mathrm{~kg}$ with width $1.01 \mathrm{~m}$, length $1.03 \mathrm{~m}$ and height $0.97 \mathrm{~m}$ (Figure 3). 


\subsection{Evaluation of the training aid}

Mechanical stability was assessed by determining the deflection and movement of the frame from video for vertical impacts using 20 - $65 \mathrm{~kg}$ masses. Deflection and movement were measured for horizontal collisions using pendulum masses of $20-65 \mathrm{~kg}$. The impact velocities were equivalent to those expected during normal use.

After mechanical stability was established, an experienced senior gymnast of height $1.60 \mathrm{~m}$ performed standard progression drills with the training aid set at $0.97 \mathrm{~m}$ (high setting) and a fully dynamic backward handspring over the training aid with and without coach support. Data were collected in a single session from performances of eight competitive gymnasts (Table 2), who had previously acquired the skill. The skill was performed both with and without the training aid, and comparisons of the four performance parameter dimensions were made using the same video imaging system noted in section 2.1 above.

Table 2. Summary of gymnast heights and training aid settings for evaluation phase

\begin{tabular}{|c|c|c|c|c|}
\hline gymnast & $\begin{array}{c}\text { standing } \\
\text { height [m] }\end{array}$ & $\begin{array}{c}\text { skill height } \\
{[\mathrm{m}]}\end{array}$ & $\begin{array}{c}\text { predicted } \\
\text { setting }\end{array}$ & $\begin{array}{c}\text { selected } \\
\text { setting }\end{array}$ \\
\hline 1 & 1.29 & 0.74 & low & low \\
2 & 1.43 & 0.76 & medium & medium \\
3 & 1.46 & 0.85 & medium & medium \\
4 & 1.55 & 0.78 & high & medium \\
5 & 1.58 & 0.96 & high & high \\
6 & 1.60 & 0.91 & high & high \\
7 & 1.65 & 1.01 & high & high \\
8 & 1.71 & 1.00 & high & high \\
\hline
\end{tabular}

Height settings: low: $0.57 \mathrm{~m}$, medium: $0.77 \mathrm{~m}$, high: $0.97 \mathrm{~m}$.

A former experienced gymnast performed backward handsprings with the training aid on five occasions over a period of 10 weeks to assess whether there was any change in technique over time in using the aid. Figure 6 shows the training aid in use by this former gymnast.

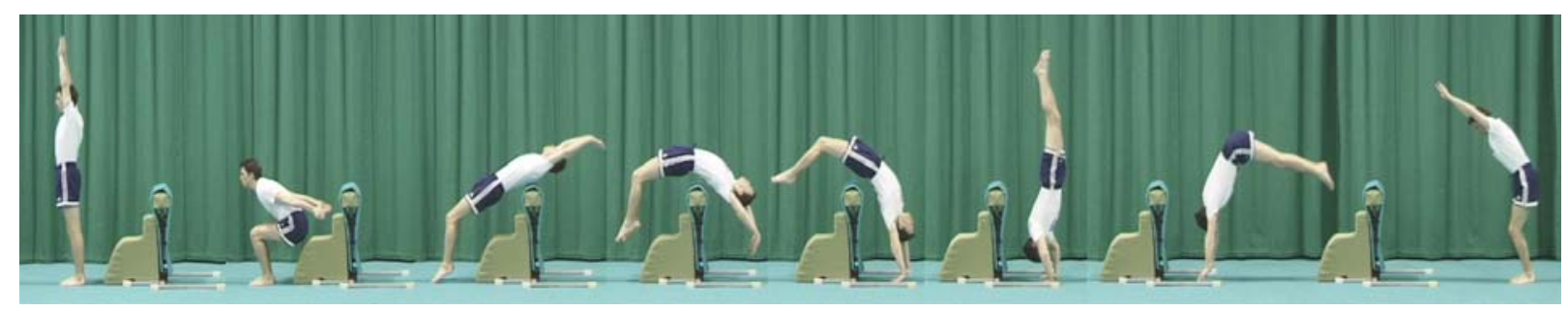

Figure 6. A backward handspring over the training aid by an experienced gymnast. 
Finally, the use of the training aid with novice gymnasts who had not acquired the skill fully was assessed. During this phase of the study only two gymnasts were analysed and so the results from this part of the study may be more subject-specific due to the limited numbers involved. The two gymnasts carried out this training alongside a group of nine other gymnasts who did not have access to the training aid. Gymnast A, an eight year old female regional level gymnast, was at the stage in her training where she needed to learn a backward handspring but had performed only basic shaping and dive-back preparations within her training sessions to this point. She learned the skill from the beginning using the training aid for 7.5 hours over a 12 week period. Gymnast B, a nine year old female club level gymnast, had taught herself a backward handspring before joining a gymnastics club, but her performance was poor (International Judge deductions in excess of 1.0) and potentially dangerous. Gymnast B used the training aid (for 7 hours over a 10 week period) to relearn the skill and improve her poor take-off technique in which her knees travelled forward reducing the horizontal velocity of her centre of mass leading to collapse on landing (Figure 8a).

The coach of gymnasts A and B was interviewed after using the training aid for 12 weeks to assess the functionality and ease of use of the prototype in comparison with existing training aids. These comprised: a cylinder over which the gymnast arches through handstand, a $3 / 4$ cylinder in which the gymnast sits and rolls to pass through handstand, an inclined mat from which the gymnast performs a backward handspring downhill, and a harness which is held by coaching assistants and provides support. The coach was questioned on the effectiveness of the support the aid provided to the gymnasts, specifically during progressive drills and poor full performances of the skill. The coach was also asked on the effect the aid had on the rate of learning, if it produced confidence in the gymnasts, if it was used without additional coach support, and if the aid resulted in additional training attempts for the gymnasts beyond their standard training. This was only an assessment of the training aid by one coach and should be viewed as a single case study.

\section{Results and Discussion}

\subsection{Mechanical stability}

The completed prototype (Figures 3 and 6), when loaded vertically as in section 2.3 gave deflection and movement values of $0 \mathrm{~mm}$ and $0^{\circ}$ respectively. For horizontal impacts the deflection and rotation were $0 \mathrm{~mm}$ and $5^{\circ}$ respectively. These low values indicated that the training aid was sufficiently rigid and stable for gymnasts up to mass $65 \mathrm{~kg}$ to use safely.

\subsection{Use by skilled gymnasts}

Gymnasts $1-8$ trialled the training aid settings for their heights and one gymnast preferred a lower setting (Table 2). The performances of these gymnasts with the training aid (supported) and without the training aid (unsupported) were compared based on the four performance parameters obtained from the video data. These data showed the following general trends: the sit height values remained very similar for the supported and unsupported performances for the majority of the gymnasts, the mean values lying within only $1.9 \%$ of each other; the sit length values showed a similar pattern, with the mean values lying within $2.4 \%$ of each other; skill length increased for all gymnasts but one, with an average increase of $25.6 \%$; skill height increased for every gymnast, with an average increase of $22.9 \%$ (Figure 7). These trends suggest that the seat section of the training aid did not interfere physically with the gymnasts; the presence of the upright section, however, encouraged a longer and higher skill. All eight gymnasts were able to perform a backward handspring over the 
training aid which did not physically interfere with these performances. It was apparent, however, that some gymnasts were attempting to clear the training aid as an obstacle rather than using their normal technique, indicating a psychological effect on the gymnasts from the presence of the training aid. For these gymnasts, this was their first experience of practising the skill over a training aid which lead to a tendency to ensure that the upright was cleared. This lessened with familiarity, as shown by the former gymnast who replicated his normal unaided performance (Figure 1) by the end of the 10 week use period, when he made only brushing contact with the training aid (Figure 6).

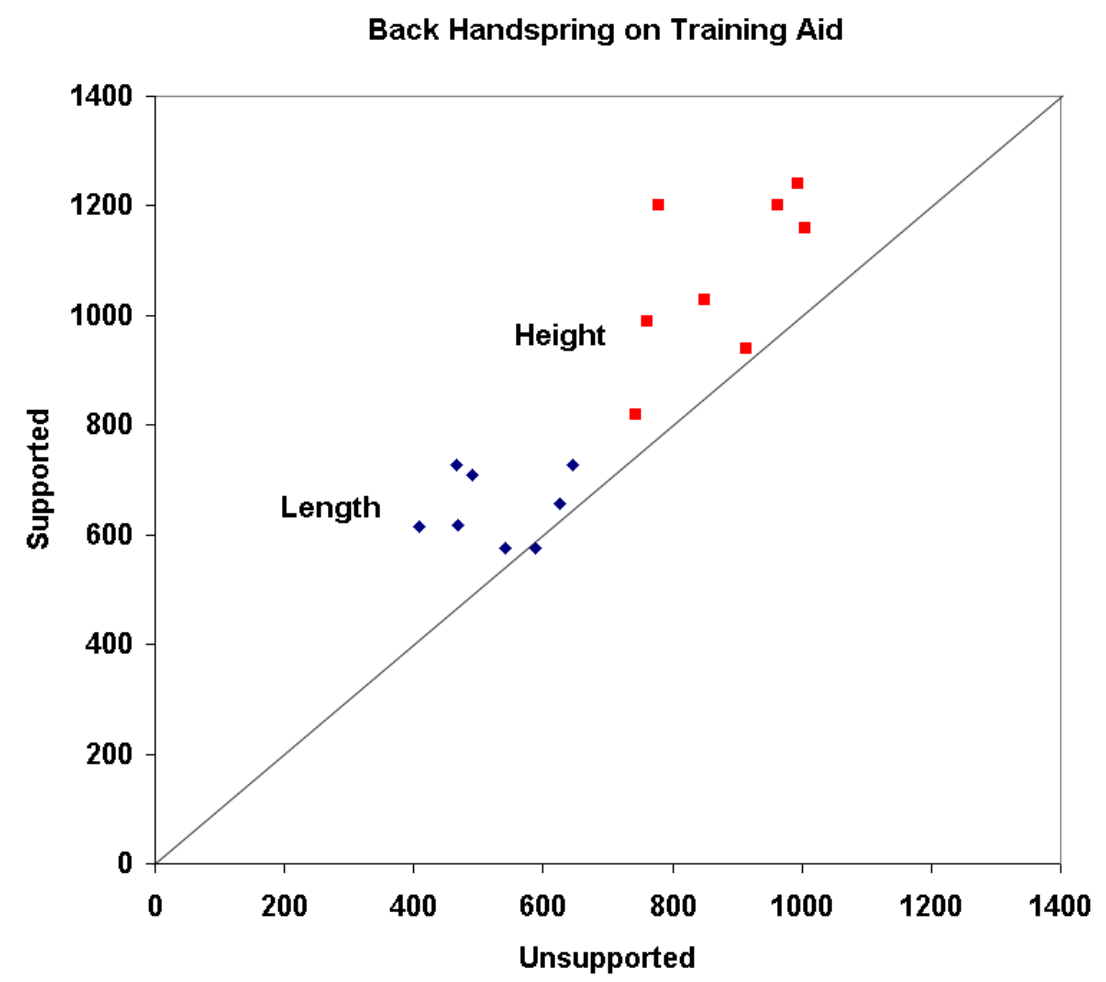

Figure 7. Skill height $(\mathrm{mm})$ and skill length $(\mathrm{mm})$ comparisons for performances of a backward handspring for supported (y-axis) against unsupported (x-axis) by the training aid.

\subsection{Use of the training aid for gymnasts acquiring the skill}

After seven and a half hours practice over a period of 12 weeks the novice gymnast A was able to perform a backward handspring safely without support. Although not perfect, she demonstrated good technique and was reported (by interview with the coach) to be confident in using the prototype without coach support. This gymnast had progressed faster in acquiring this skill than the rest of the group.

Gymnast B was also reported to be confident in using the prototype without coach support and after less than seven hours training spread over 10 weeks, which included time to accustom herself to the new training aid, the backward handspring technique had improved considerably. Gymnast B no longer pushed her knees forward during takeoff, her takeoff direction and first flight had improved, and consequently she was now capable of landing the skill on her feet (Figures 8a and b). This improvement in technique also occurred at a faster rate than the rest of the gymnast's training group. 
(a) before training

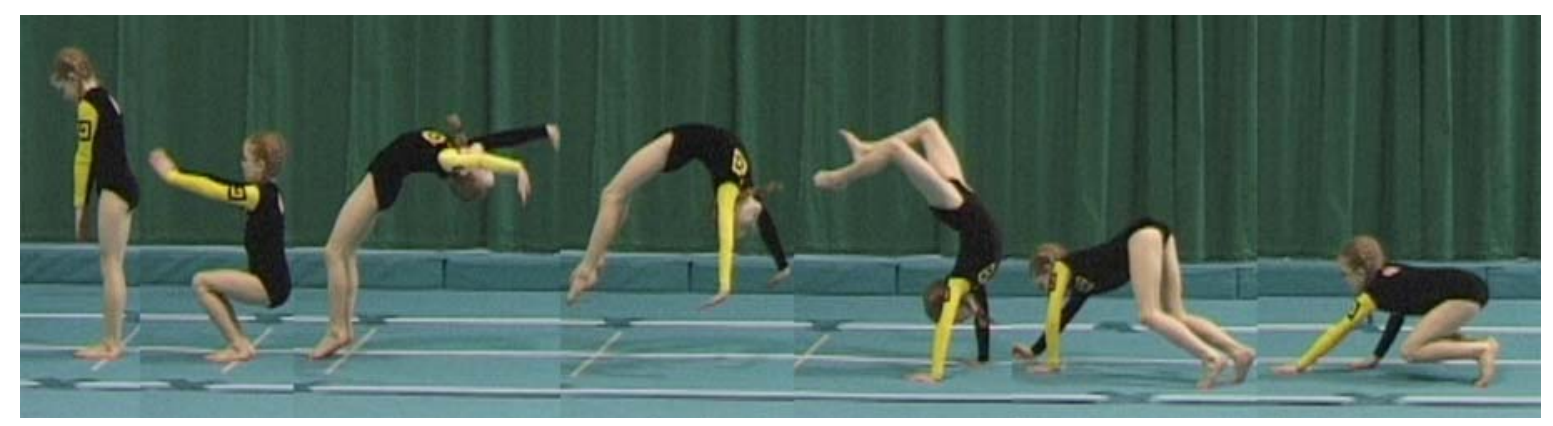

(b) after training

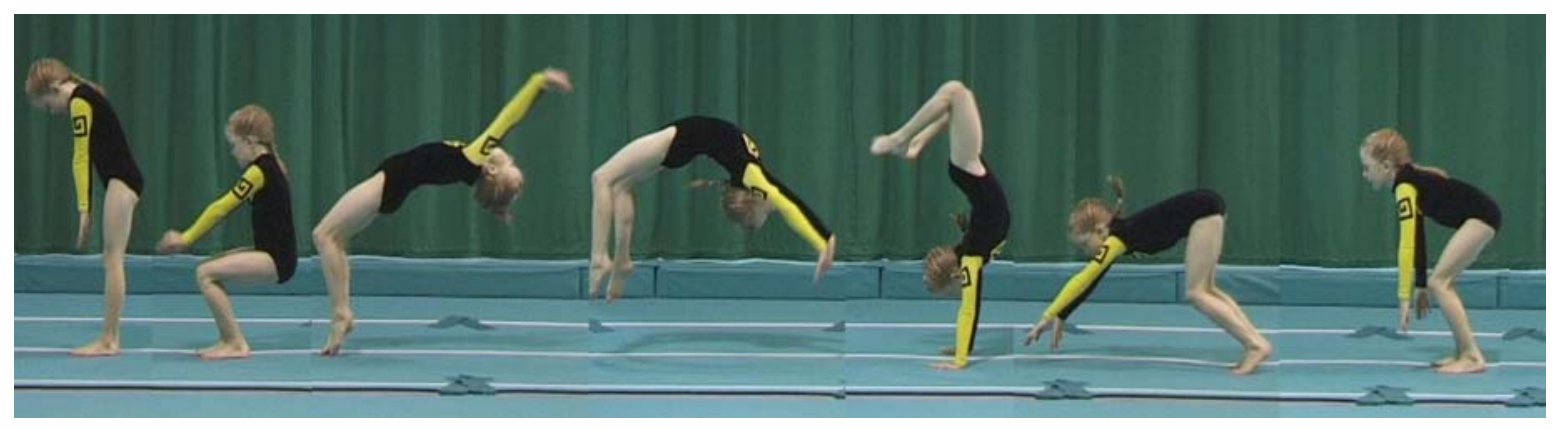

Figure 8 . Performances of a backward handspring by gymnast B: (a) before training and (b) after training.

The training aid was intended to be used by inexperienced gymnasts rather than by experienced gymnasts who had already mastered the skill. Once an inexperienced gymnast became accustomed to the aid, it was found to encourage good technique through increased take-off power and trajectory. When the trajectory was low, the gymnast would not clear the height of the aid. When the trajectory was high and short, the gymnast would generally land on the top of the aid. During performances with diminished power, the aid supported the lack of flight and was observed to prevent the gymnasts landing awkwardly or in a dangerous orientation. Neither of the inexperienced novice gymnasts who learned the skill using the training aid attempted to clear the training aid using a long and high backward handspring, which would be consistent with increased familiarity in making contact with the training aid which had supported their inadequate efforts at times. Through this interaction with the prototype, the two gymnasts gained confidence to repeatedly perform the skill with the power, height and length required to 'clear' the aid when set up for their height. The training aid allowed increased technically correct repetition and provided crucial support in the training sessions, allowing both gymnasts to progress at a higher rate than the remainder of the group. These assessments are the opinion of the coach who used the training aid and should be regarded as a single case study.

\subsection{Comparison with other training aids}

The assessment of training aids shown in Table 3 indicates that the prototype aid fulfilled all of the coaching and design requirements whereas the other aids were deficient in at least three of the eight requirements. Although limited in extent, this study revealed that it was possible to use the training aid for a range of standard progression drills, and the 
adjustability of the device allowed for the drills to be performed with a variety of levels of support. The prototype training aid could be used by gymnasts without coach support, which was not possible with the other training aids.

Table 3. Assessment of training aids using key coaching requirements

\begin{tabular}{|c|c|c|c|c|c|c|c|c|}
\hline $\begin{array}{l}\text { Training } \\
\text { Aid }\end{array}$ & 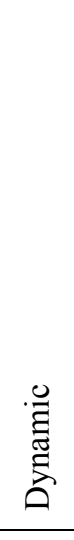 & 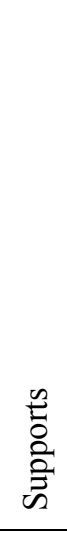 & 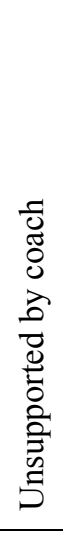 & 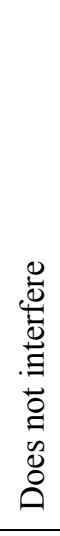 & 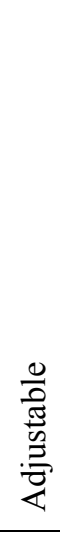 & 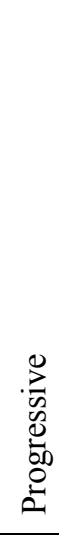 & 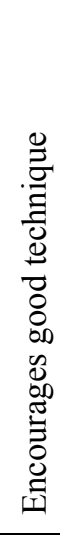 & 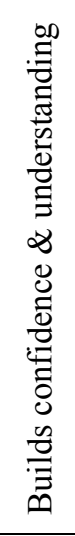 \\
\hline Cylinder & $x$ & $\checkmark$ & $x$ & $x$ & $x$ & $\checkmark$ & $x$ & $\checkmark$ \\
\hline $\begin{array}{l}3 / 4 \\
\text { Cylinder }\end{array}$ & $x$ & $x$ & $x$ & $x$ & $x$ & $\checkmark$ & $x$ & $\checkmark$ \\
\hline Incline & $\checkmark$ & $x$ & $x$ & $\checkmark$ & $x$ & $x$ & $\checkmark$ & $\checkmark$ \\
\hline Harness & $\checkmark$ & $\checkmark$ & $x$ & $\checkmark$ & $\checkmark$ & $x$ & $x$ & \\
\hline Prototype & $\checkmark$ & $\checkmark$ & $\checkmark$ & $\checkmark$ & $\checkmark$ & $\checkmark$ & $\checkmark$ & \\
\hline
\end{tabular}

\section{Conclusions and Further Work}

A prototype training aid was designed, manufactured and tested. It permitted a safe dynamic performance and provided support but did not obstruct technically good performances; could be used without coach support and was shown to be adjustable for gymnasts of various heights between $1.1 \mathrm{~m}$ and $1.7 \mathrm{~m}$ through the use of the nine experienced gymnasts and the two novice gymnasts. The aid allowed progressive practice and encouraged good technique in that it provided support for low or short attempts. The training aid appeared to increase the rate at which inexperienced gymnasts improved their technique. After gaining familiarity with the device an experienced gymnast was able to reproduce his normal technique when performing a backward handspring over the training aid.

The evaluation of the training aid using two case studies is obviously limited by the low number of participants. A more comprehensive evaluation might comprise a comparison of the performance of a group learning the backward handspring using the training aid with another group who received coach support during a session of equal time. While such studies are rare they are a necessary part of any scientific assessment of coaching practice.

\section{Acknowledgements}

We would like to thank John White of Gymnova for his support in developing the prototype training aid. 


\section{References}

Gervais, P., Wu, T., \& LeBlanc, J.S., (2004) A Comparison of Three Back Handspring Progressions. In: Proceedings of the 13th Biennial Conference of the Canadian Society for Biomechanics, Halifax, Nova Scotia, pp. 118.

Hayhurst, B., (1980) The standing back-flick. The Gymnast, 17(9), 8-9.

Holding, D.H., (1989) Skills Research. In: Human Skills (ed D. Holding), pp. 1-16. John Wiley and Sons, Chichester.

Koh, T.J., Grabiner, M.D., \& Weiker, G.G., (1992) Technique and ground reaction forces forces in the back handspring. American Journal of Sports Medicine 20(1), 61-66.

Mitchell, D., Davis, B. \& Lopez, R., (2002) Teaching Fundamental Gymnastics Skills, pp. 108-176. Human Kinetics, Champaign, IL.

Sands, W.A. \& McNeal, J.R., (2006) Hand position in a back handspring (flic flac). Technique, 26(3) 8-9. 